Research Article

\title{
Flexible, Transparent, and Conductive Film Based on Random Networks of Ag Nanowires
}

\author{
Shunhua Wang, Xu Zhang, and Weiwei Zhao \\ School of Mechatronic Engineering, Lanzhou Jiaotong University, Lanzhou 730070, China \\ Correspondence should be addressed to Xu Zhang; zhangxu20054125@163.com
}

Received 15 March 2013; Accepted 22 March 2013

Academic Editor: Tifeng Jiao

Copyright ( 2013 Shunhua Wang et al. This is an open access article distributed under the Creative Commons Attribution License, which permits unrestricted use, distribution, and reproduction in any medium, provided the original work is properly cited.

\begin{abstract}
Flexible, transparent, and conductive films based on random networks of Ag nanowires were prepared by vacuum-filtrating method. The size of Ag nanowires prepared by hydrothermal method is uniform, with a relatively smaller diameter and a longer length, thereby achieving a high aspect ratio (>1000). The films fabricated by Ag nanowires exhibit the excellent transparency with a $92 \%$ optical transmittance and a low surface resistivity of $11 \Omega \mathrm{sq}^{-1}$. Importantly, both the transmittance and sheet resistance decrease with the increasing of the Ag nanowires contents. When the contents of $\mathrm{Ag}$ nanowires are up to $200 \mathrm{mg} / \mathrm{m}^{2}$ especially, the surface resistivity quickly falls below $5 \Omega \mathrm{sq}^{-1}$. Also, these films are robust, which have almost no change in sheet resistance after the repeating bends over 200 cycles. These encouraging results may have a potential application in flexible and transparent electronics and other heating systems.
\end{abstract}

\section{Introduction}

Two-dimensional electrothermal heating elements (films), especially transparent and flexible film heaters, have attracted growing interest for a wide range of applications including outdoor displays, vehicle window defrosters, heating retaining windows, and other heating systems [1]. Typically, indium tin oxide (ITO) has been used as transparent conductive films due to its excellent transparency throughout the visible spectrum, low sheet resistance, and the compatibility of its work function with the injection and collection of charge carriers in organic semiconductors. However, ITO has certain limitations in application for next-generation devices: (i) the increasing price due to indium scarcity and processing requirements, which renders it difficult to use in low-cost, large-area electronics; (ii) ITO is brittle and can easily wear out or crack when used in applications where bending is involved [2-4]. To address these issues, recent works have devised strategies based on materials that have potential for large-area coverage and some degree of mechanical compliance. These materials include conducting polymers $[5,6]$, carbon nanotubes (CNTs) [7, 8], graphenes [9-11], metal grids [12], and metallic nanowires [13-16]. The performance of these materials for applications as transparent films is largely governed by two critical parameters: the surface resistivity and the optical transmittance. However, achieving low surface resistivity and high optical transmittance is still a challenge because these two values follow unfavorably opposing trends, and this often results in optoelectronic performances far inferior to those of ITOs. Promisingly, Ag nanowires (AgNWs) with high electrical conductivity $\left(\sim 6.3 \times 10^{7} \mathrm{~S} / \mathrm{m}\right)$ are considered as candidates for using in flexible electronics [17]. Transparent electrodes composed of random AgNWs networks can be readily achieved by simple and scalable solution processing such as spin coating [18], rod coating [19], drop casting [20, 21], and air spraying [22] from an AgNWs dispersion. Such application of AgNWs has been reported by many groups, including the possibilities for its use as transparent electrodes in optoelectronic applications [10-17]. De et al. demonstrated a method of producing AgNW films with an optical transmittance $(T)$ of $\approx 85 \%$ and surface resistivity $\left(R_{s}\right)$ of $13 \Omega \mathrm{sq}^{-1}$ [23]. Lee et al. reported solution-processed AgNW films with $T=80 \%$ and $R_{s}=20 \Omega \mathrm{sq}^{-1}$ [21]. Scardaci et al. demonstrated that AgNWs can be spray deposited over large areas to form networks with $T=90 \%$ and $R_{s}=50 \Omega \mathrm{sq}^{-1}$ [22].

In this work, we present a method to prepare highly transparent, conductive, and flexible AgNWs-based films. 
The solution-based synthetic method was used to prepare AgNWs, and then vacuum filtrating of AgNWs aqueous dispersions was employed to prepare transparent electrode, through which the nanowire networks are efficiently interconnected, with optical transmittance and sheet resistance of $92 \%$ and $11 \Omega \mathrm{sq}^{-1}$, respectively. Furthermore, we explore the use of uniform AgNWs networks as a transparent electrode for film heaters, which show a better performance than ITO films in terms of mechanical flexibility and rapid thermal responses. There are, however, several technical issues associated with their practical application, including the scalable processes for fabricating large-scale film heaters and the low operation voltage required for automobile window defrosters [24-26]. Here, we demonstrate that optimally engineered AgNWs-based films outperform other transparent conducting materials in that they show outstanding optical and electrical properties and enable rapid heating at low input voltages.

\section{Experimental}

In this paper, Ag nanowires were synthesized by a solvothermal method [27] with a modification. Typically, $0.544 \mathrm{~g}$ $\mathrm{AgNO}_{3}$ was dissolved in $48 \mathrm{~mL}$ of ethylene glycol (EG, 99\%), and $1.4128 \mathrm{~g}$ polyvinylpyrrolidone (PVP, MW $=1.3 \mathrm{Mg} / \mathrm{mol}$ ), and $3.68 \mathrm{mg} \mathrm{NaCl}$ was dissolved in $32 \mathrm{~mL} \mathrm{EG}$ at $120^{\circ} \mathrm{C}$ with continuous stirring at $600 \mathrm{rpm}$. Subsequently, the $\mathrm{PVP} / \mathrm{NaCl} / \mathrm{EG}$ solution was added to the $\mathrm{AgNO}_{3} / \mathrm{EG}$ solution drop by drop under vigorous stirring. The combined solution was allowed to mix for $5 \mathrm{~min}$ and was then transferred into a $100 \mathrm{~mL}$ autoclave. The autoclaves were heated at $160^{\circ} \mathrm{C}$ for $7 \mathrm{~h}$ and then cooled to room temperature. The final dispersion was diluted with methanol and centrifuged at $4000 \mathrm{rpm}$ for $30 \mathrm{~min}$. After centrifugation, the supernatant dissolved residual EG was removed by syringe. Then, methanol was added into the centrifuge tube to disperse the products and to dissolve the residual PVP. After centrifugation, the supernatant was also removed by syringe, and the process was repeated twice more. The resulting silver nanowires were dispersed into ethanol to $1 \mathrm{mg} / \mathrm{mL}$.

A dilute AgNWs suspension was vacuum filtrated using a Millipore filter ( $50 \mathrm{~mm}$ in diameter and $0.45 \mu \mathrm{m}$ in pore size). The membranes with captured film were then cut into size of choice, wetted with deionized water, and transferred on to poly(ethylene terephthalate) (PET) or quartz glass using isopropanol to remove trapped air between the films and the substrate thus improving adhesion. The cellulose filter membrane was dissolved using acetone (successively pure baths) to leave a thin film on the substrate. The film was then rinsed with a methanol wash and dried at room temperature.

The morphology and microstructure of the as-prepared AgNWs and films were investigated using a field emission scanning electron microscope (FESEM, JSM6701F). The optical transmission spectra of the AgNW films were recorded using a UV-vis spectrometer (Varian Cary 5000). In all cases, a quartz glass slide was used as the reference. The sheet resistance measurements were made using the four-probe technique (MCP-T610).
An AgNWs-based film heater on quartz glass $(25 \mathrm{~mm}$ $\times 25 \mathrm{~mm}$ ) was made in a two-terminal side contact configuration. The DC voltage was supplied by a power supply (Su Zhou Varied Electronics Co., LTD) to the film heaters through a copper or silver contact at the film edge; a digital multimeter was used to measure the average surface temperature of the films in real time by a thermal couple.

\section{Results and Discussion}

Figure 1(a) shows the field emission scanning electron microscope (FESEM) images of Ag nanowires (AgNWs). The modified solvothermal synthetic method makes the AgNWs with a relatively smaller diameter and a longer length, thereby achieving a high aspect ratio $(>1000)$. The diameter of assynthesized AgNWs is $40-90 \mathrm{~nm}$ and of lengths up to 40$90 \mu \mathrm{m}$. The inset of Figure 1(a) is the photograph of AgNWs aqueous dispersions, and the solution is stable and homogeneous.

The highly transparent, conductive, and flexible AgNWsbased electrodes were prepared by depositing AgNWs onto a cellulose membrane by vacuum filtration. Then, the film was attached to the PET substrate or quartz glass using isopropanol and pressure and the cellulose dissolved in acetone. The density of the AgNWs networks can be easily controlled by adjusting the concentration of aqueous dispersions. Figure 1(b) presents FESEM image of AgNWs-based film. The AgNWs constitute an efficient electrical conduction path across the film without a significant loss of optical transmittance. It is clearly seen from Figures $1(\mathrm{c})$ and 1(d) that the AgNWs-based film which transferred on to quartz glass (the left is a black quartz glass as the reference) or PET appears to be of very high optical transparence.

As shown in Figures 2(a) and 2(b), the optical transmittance and surface resistivity of the AgNWs-based film on the quartz glass substrates are measured as a function of the AgNW s contents. With the increase of AgNWs contents, both the surface resistivity and optical transmittance decrease. At a wavelength of $550 \mathrm{~nm}$, the optical transmittance values of the AgNWs-based electrodes with AgNWs contents of $40 \mathrm{mg} / \mathrm{m}^{2}, 80 \mathrm{mg} / \mathrm{m}^{2}, 200 \mathrm{mg} / \mathrm{m}^{2}$, and $400 \mathrm{mg} / \mathrm{m}^{2}$ are 96.1 , 91.7, 44.3, and 34.8\%, respectively. All of the AgNWs-based electrodes are transparent so that our university logo is clearly visible through the electrodes (Figure 1(c)). The surface resistivity of the AgNWs-based electrodes with AgNWs contents of $40 \mathrm{mg} / \mathrm{m}^{2}, 80 \mathrm{mg} / \mathrm{m}^{2}, 200 \mathrm{mg} / \mathrm{m}^{2}$, and $400 \mathrm{mg} / \mathrm{m}^{2}$ is 31,10 , 5 , and $2 \Omega \mathrm{sq}^{-1}$. As shown in Figure 2(b), the AgNWs-based electrodes with AgNWs contents of $40 \mathrm{mg} / \mathrm{m}^{2}$ and $80 \mathrm{mg} / \mathrm{m}^{2}$ appear to be with very high optical quality and low surface resistivity, which could be fully capable as transparent conductive film heaters.

Figure 3(a) shows the time-dependent temperature profiles of the AgNWs-based film heater with respect to the surface resistivity of the AgNWs film. Under the application of the same input voltage $(5 \mathrm{~V})$, the maximum temperature at a steady state increases with the decrease of surface resistivity of the film, which suggests that the $R_{s}$ value of the AgNWs film should be less than $10 \Omega \mathrm{sq}^{-1}$ to afford the maximum temperature above $90^{\circ} \mathrm{C}$ at an input voltage of 5 V. Figure 3(b) 


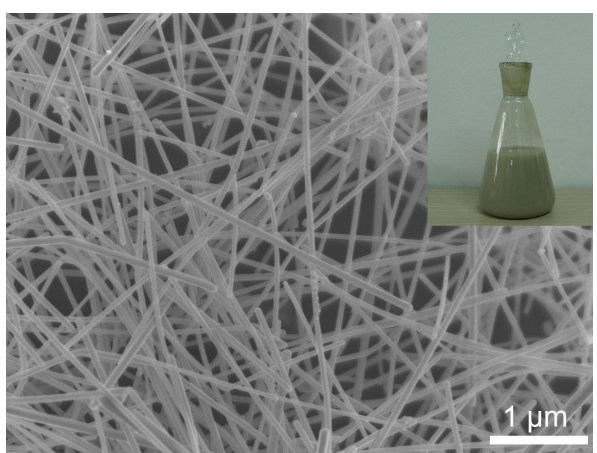

(a)

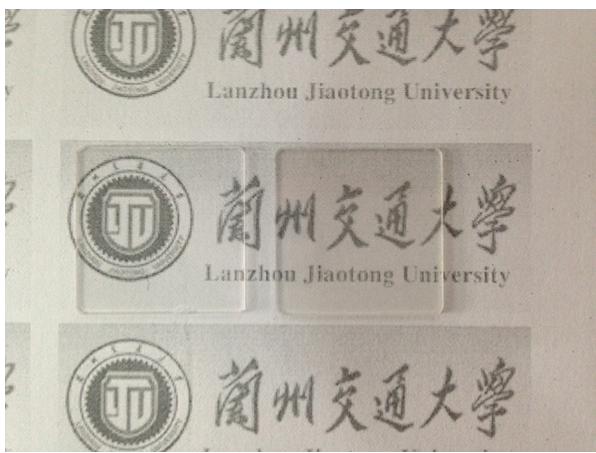

(c)

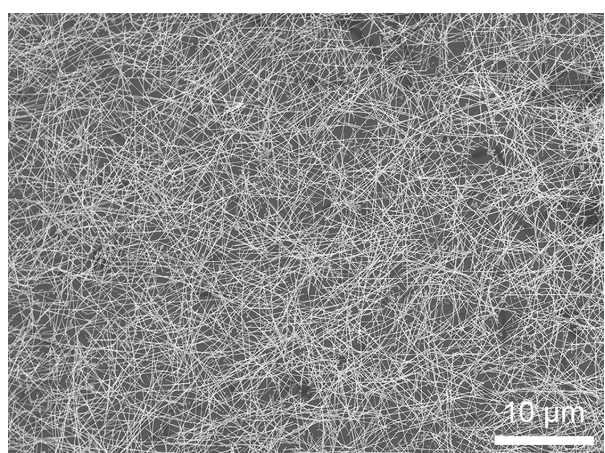

(b)

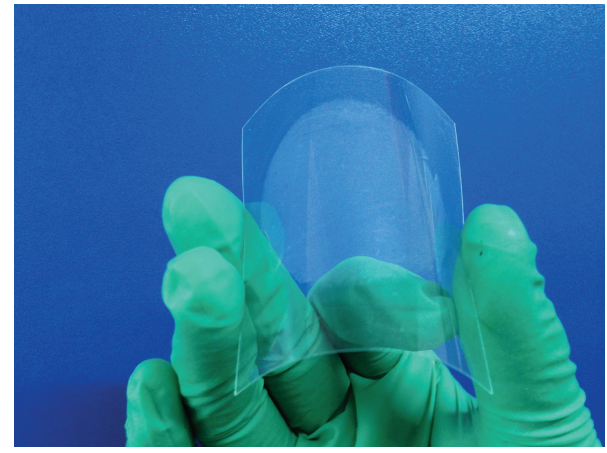

(d)

FIGURE 1: FESEM images of AgNWs (a) and film (b), optical images after Ag nanowires deposition onto quartz glass (c), and PET substrate (d).

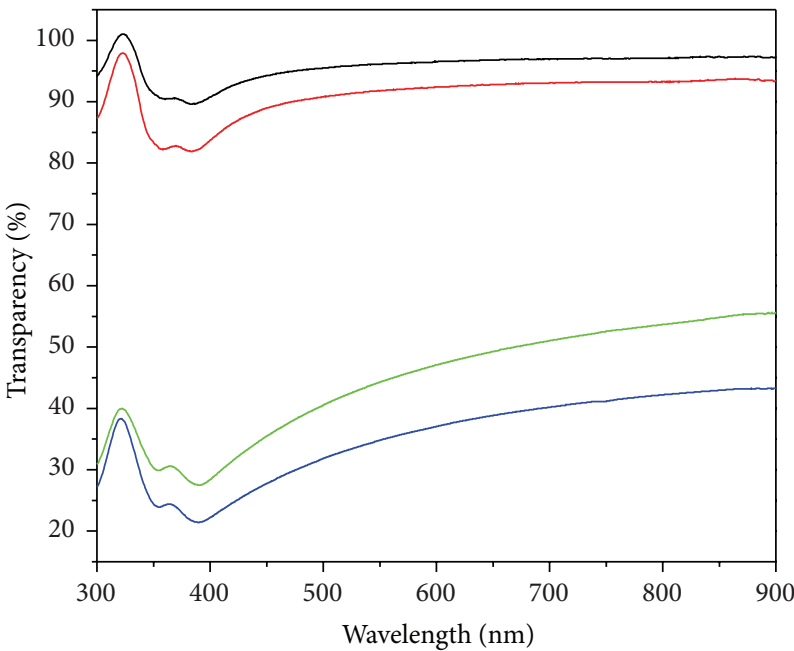

$\begin{aligned} & \text { Ag content } 40 \mathrm{mg} / \mathrm{m}^{2} \\ & \text { Ag content } 80 \mathrm{mg} / \mathrm{m}^{2}\end{aligned}-\mathrm{Ag}$ content $200 \mathrm{mg} / \mathrm{m}^{2}$

(a)

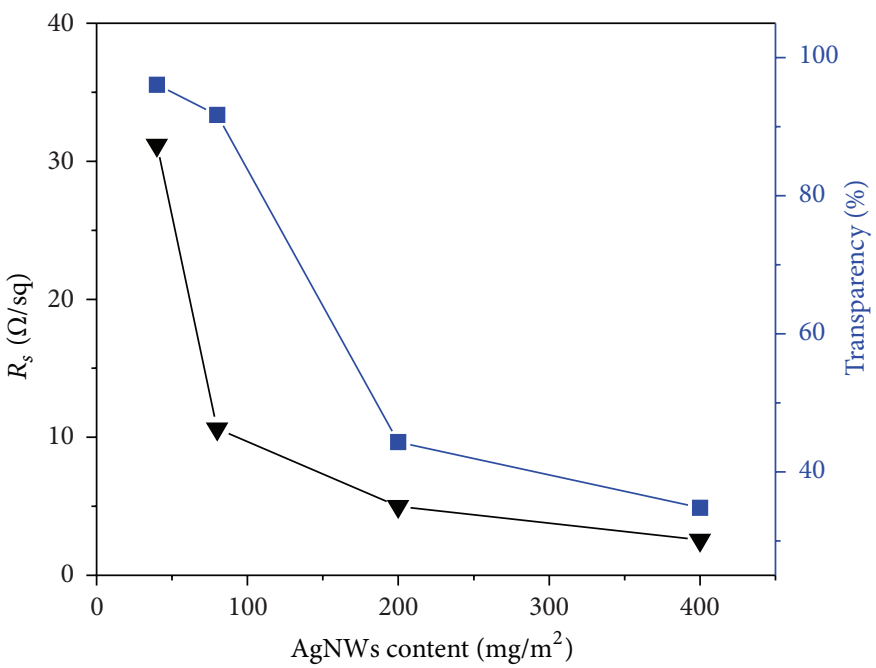

(b)

Figure 2: (a) Total and specular optical transmission of the Ag nanowires film over the wavelength of 300-900 nm and (b) plot of transmittance (at $\lambda=550 \mathrm{~nm}$ ) and sheet resistance versus Ag nanowires content for the films of AgNWs.

shows the temperature profiles of the film heater assembled with an AgNWs film (AgNWs content $80 \mathrm{mg} / \mathrm{m}^{2}$ ), which is plotted with respect to the input voltages (modulated from 3 to $7 \mathrm{~V}$ ). When the input voltage increases to $7 \mathrm{~V}$, the film heater reaches a temperature above $138^{\circ} \mathrm{C}$, which confirms its operability at low input voltages. A high power at a low input voltage implies the efficient transduction of electrical energy into Joule heating, which attributed to the improved 


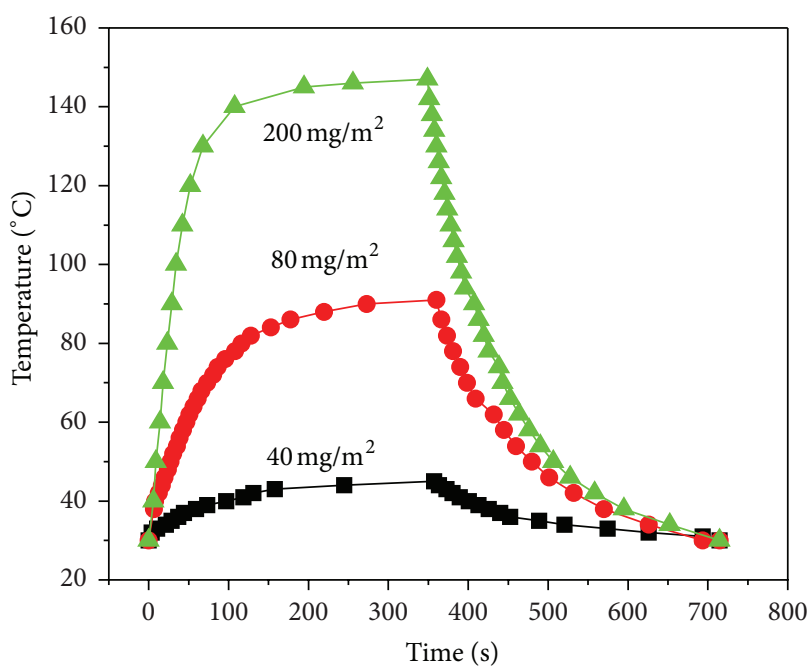

(a)

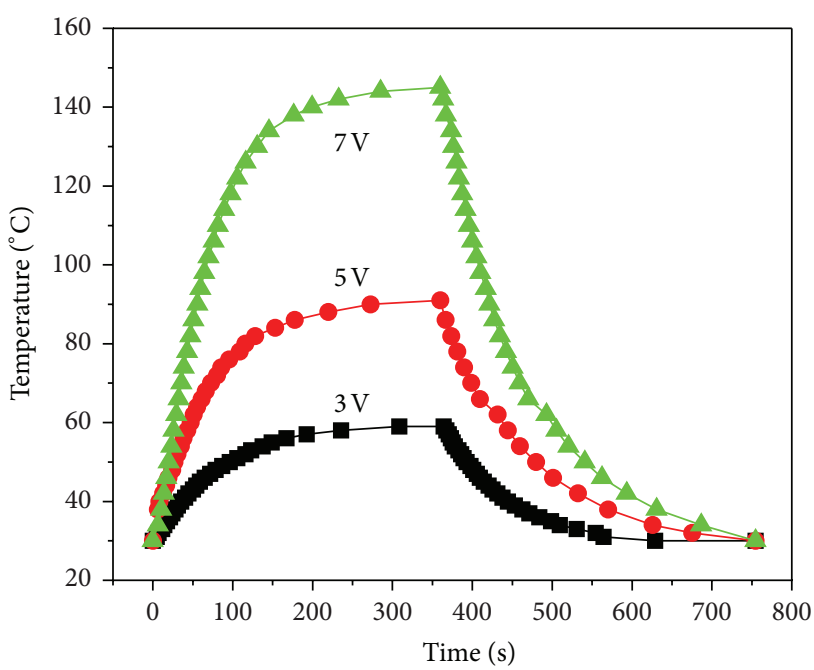

(b)

Figure 3: (a) Time-dependent temperature profiles of AgNWs-based film heaters with respect to the surface resistivity of the AgNWs films and (b) temperature profiles of the AgNWs film heater $\left(80 \mathrm{mg} / \mathrm{m}^{2}\right)$ operated at different input voltages.

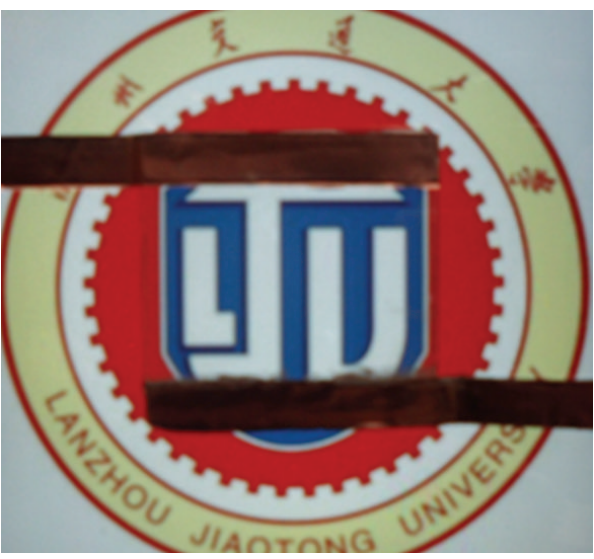

(a)

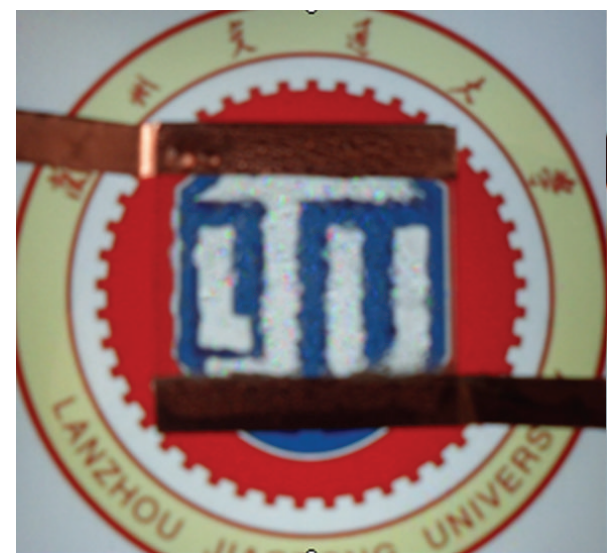

(b)

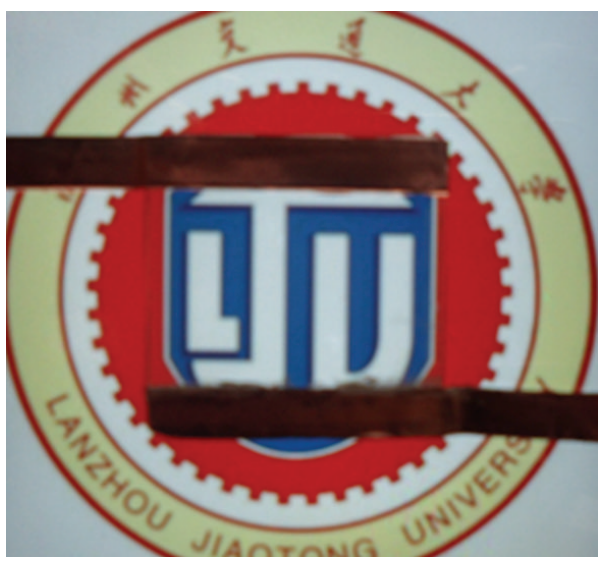

(c)

FIGURE 4: Defrosting test results of a random network of AgNWs film heater: (a) optical photo before and (b) after frost formation and (c) after operation of the film heater at $10 \mathrm{~V}$. 


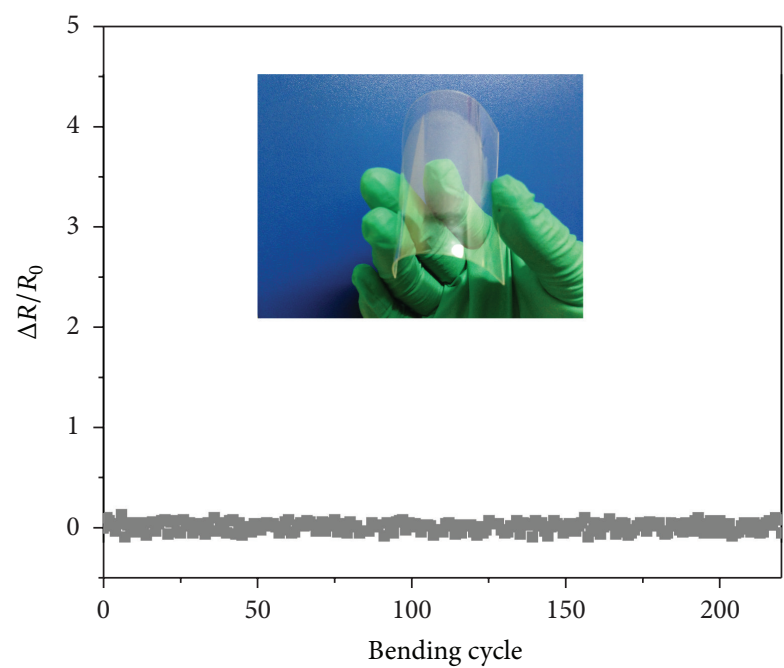

FIGURE 5: Resistance change of AgNWs film under bending testing; the inset photo shows the bending process.

conductivity of the AgNWs film. The response time, which is defined as the time required to reach the steady-state temperature, is one of the key factors for evaluating the performance of film heaters. Regardless of the input voltages, the steady-state temperature of the film heater is reached within $150 \mathrm{~s}$, demonstrating the fast response of the device. Considering that a film heater based on $\mathrm{Ag}$ nanowires reaches $70^{\circ} \mathrm{C}$ under an input voltage of $5 \mathrm{~V}$ [25], our AgNWs-based device outperforms other nanostructured material-based film heaters too and thus will be useful for applications that require fast temperature switching with low input voltages.

To further demonstrate its potential as an efficient defroster, a defrost test was performed with the AgNWsbased film heater (Figure 4). For this test, an AgNWs film was attached to a glass substrate (Figure 4(a)) and put in a refrigerator for $30 \mathrm{~min}$ to allow frost to form on the entire AgNWs film (Figure 4(b)). With the film heater operating at $10 \mathrm{~V}$, the frost on the surface was completely removed within $40 \mathrm{~s}$, making the symbol marks in the background clearly visible (Figure 4(c)). Therefore, these solution-processed AgNWsbased films may enable new application such as defrost for highly transparent large-scale film heaters.

Another advantage of our AgNWs-based film heater is the mechanical robustness against adhesion and bending, since the AgNWs are firmly anchored on PET films. For this purpose, we investigated the effect of bending on the electrical resistance of the AgNWs film with $R_{s} \approx 10 \Omega \mathrm{sq}^{-1}$ and $T \approx 92 \%$. The bending test was performed in which the films were repeatedly bent at a radius of curvature of $10 \mathrm{~mm}$. Figure 5 inset shows a photograph of the AgNWs film on PET substrate, demonstrating its flexibility and transparency. The resistance variations obtained during the repetitive bending are represented by the $R-R_{0}=\Delta R / R_{0}$, where $R_{0}$ is the initial sheet resistance and $R$ is the sheet resistance measured when flattened after a certain number of bending cycles. As shown in Figure 5 , no obvious increase in $\Delta R / R_{0}$ was observed even after 200 bending cycles. Unlike an ITO-based film heater
[28], the absence of cracking or tearing suggests high levels of mechanical flexibility of the AgNWs-based film heaters.

\section{Conclusions}

AgNWs-based electrodes with excellent heating performance and flexibility are fabricated through vacuum filtrating an AgNWs solution and subsequently transferred onto quartz and PET. Moreover, their electrothermal performances were studied in terms of heating rate and applied voltage. Heating films deposited on quartz show high transparencies and good heating effects. Defrosting experiment of the films was carried out, and remarkable efficiencies were observed. Flexible AgNWs-based films formed on PET showed exciting electrical property and robust endurance against repeated bending. As a consequence of their mechanical flexibility, high transparency and conductivity, and scalable production through easy solution processing, AgNWs-based films are excellent potential candidates for applications in electrical devices.

\section{Acknowledgment}

This work is financially supported by Program for Science and Technology Support Foundation of Lanzhou Jiaotong University (Grant no. ZC2012001).

\section{References}

[1] Z. Wu, Z. Chen, X. Du et al., "Transparent, conductive carbon nanotube films," Science, vol. 305, no. 5688, pp. 1273-1276, 2004.

[2] A. Kumar and C. Zhou, "The race to replace tin-doped indium oxide: which material will win?" ACS Nano, vol. 4, no. 1, pp. 11$14,2010$.

[3] D. S. Hecht, L. Hu, and G. Irvin, "Emerging transparent electrodes based on thin films of carbon nanotubes, graphene, and metallic nanostructures," Advanced Materials, vol. 23, no. 13, pp. 1482-1513, 2011.

[4] S. Pang, Y. Hernandez, X. Feng, and K. Müllen, "Graphene as transparent electrode material for organic electronics," Advanced Materials, vol. 23, no. 25, pp. 2779-2795, 2011.

[5] S. Kirchmeyer and K. Reuter, "Scientific importance, properties and growing applications of poly(3,4-ethylenedioxythiophene)," Journal of Materials Chemistry, vol. 15, no. 21, pp. 20772088, 2005.

[6] A. G. MacDiarmind, "A novel role for organic polymers (Nobel Lecture)," Angewandte Chemie International Edition, vol. 40, pp. 2581-2590, 2001.

[7] E. M. Doherty, S. De, P. E. Lyons et al., “The spatial uniformity and electromechanical stability of transparent, conductive films of single walled nanotubes," Carbon, vol. 47 , no. 10, pp. $2466-$ 2473, 2009

[8] V. Scardaci, R. Coull, and J. N. Coleman, "Very thin transparent, conductive carbon nanotube films on flexible substrates," Applied Physics Letters, vol. 97, no. 2, Article ID 023114, 2010.

[9] X. Li, Y. Zhu, W. Cai et al., "Transfer of large-area graphene films for high-performance transparent conductive electrodes," Nano Letters, vol. 9, no. 12, pp. 4359-4363, 2009.

[10] S. Bae, H. Kim, Y. Lee et al., "Roll-to-roll production of 30-inch graphene films for transparent electrodes," Nature Nanotechnology, vol. 5, no. 8, pp. 574-578, 2010. 
[11] G. Eda, G. Fanchini, and M. Chhowalla, "Large-area ultrathin films of reduced graphene oxide as a transparent and flexible electronic material," Nature Nanotechnology, vol. 3, no. 5, pp. 270-274, 2008.

[12] M. G. Kang, M. S. Kim, J. Kim, and L. J. Guo, "Organic solar cells using nanoimprinted transparent metal electrodes," Advanced Materials, vol. 20, pp. 4408-4413, 2008.

[13] S. De, P. J. King, P. E. Lyons, U. Khan, and J. N. Coleman, "Size effects and the problem with percolation in nanostructured transparent conductors," ACS Nano, vol. 4, no. 12, pp. 70647072, 2010.

[14] H. Wu, L. Hu, M. W. Rowell et al., "Electrospun metal nanofiber webs as high-performance transparent electrode," Nano Letters, vol. 10, no. 10, pp. 4242-4248, 2010.

[15] L. Hu, H. S. Kim, J. Y. Lee, P. Peumans, and Y. Cui, "Scalable coating and properties of transparent, flexible, silver nanowire electrodes," ACS Nano, vol. 4, no. 5, pp. 2955-2963, 2010.

[16] V. Scardaci, R. Coull, P. E. Lyons, D. Rickard, and J. N. Coleman, "Spray deposition of highly transparent, low-resistance networks of silver nanowires over large areas," Small, vol. 7, pp. 2621-2628, 2011.

[17] C. H. Liu and X. Yu, "Silver nanowire-based transparent, flexible, and conductive thin film," Nanoscale Research Letters, vol. 6, article 75, 2011.

[18] D. S. Leem, A. Edwards, M. Faist, J. Nelson, D. D. Bradley, and J. C. de Mello, "Efficient organic solar cells with solutionprocessed silver nanowire electrodes," Advanced Materials, vol. 23, pp. 4371-4375, 2011.

[19] A. R. Madaria, A. Kumar, F. N. Ishikawa, and C. Zhou, "Uniform, highly conductive, and patterned transparent films of a percolating silver nanowire network on rigid and flexible substrates using a dry transfer technique," Nano Research, vol. 3, no. 8, pp. 564-573, 2010.

[20] J. Y. Lee, S. T. Connor, Y. Cui, and P. Peumans, "Semitransparent organic photovoltaic cells with laminated top electrode," Nano Letters, vol. 10, no. 4, pp. 1276-1279, 2010.

[21] J. Y. Lee, S. T. Connor, Y. Cui, and P. Peumans, "Solution-processed metal nanowire mesh transparent electrodes," Nano Letters, vol. 8, no. 2, pp. 689-692, 2008.

[22] V. Scardaci, R. Coull, P. E. Lyons, D. Rickard, and J. N. Coleman, "Spray deposition of highly transparent, low-resistance networks of silver nanowires over large areas," Small, vol. 7, pp. 2621-2628, 2011.

[23] S. De, T. M. Higgins, P. E. Lyons et al., "Silver nanowire networks as flexible, transparent, conducting films: extremely high DC to optical conductivity ratios," ACS Nano, vol. 3, no. 7, pp. 17671774, 2009.

[24] Y. H. Yoon, J. W. Song, D. Kim et al., "Transparent film heater using single-walled carbon nanotubes," Advanced Materials, vol. 19, no. 23, pp. 4284-4287, 2007.

[25] T. Kim, Y. Kim, H. Lee, H. Kim, W. Yang, and K. S. Suh, "Uniformly interconnected silver-nanowire networks for transparent film heaters," Advanced Functional Materials, vol. 23, pp. 1250-1255, 2013.

[26] D. Sui, Y. Huang, L. Huang et al., "Flexible and transparent electrothermal film heaters based on graphene materials," Small, vol. 7, pp. 3186-3192, 2011.

[27] W. C. Zhang, X. L. Wu, H. T. Chen et al., "Self-organized formation of silver nanowires, nanocubes and bipyramids via a solvothermal method," Acta Materialia, vol. 56, no. 11, pp. 25082513, 2008.
[28] A. Kim, Y. Won, K. Woo, C. H. Kim, and J. Moon, "Highly transparent low resistance $\mathrm{ZnO} / \mathrm{Ag}$ nanowire/ZnO composite electrode for thin film solar cells," ACS Nano, vol. 7, no. 2, pp. 10811091, 2013. 

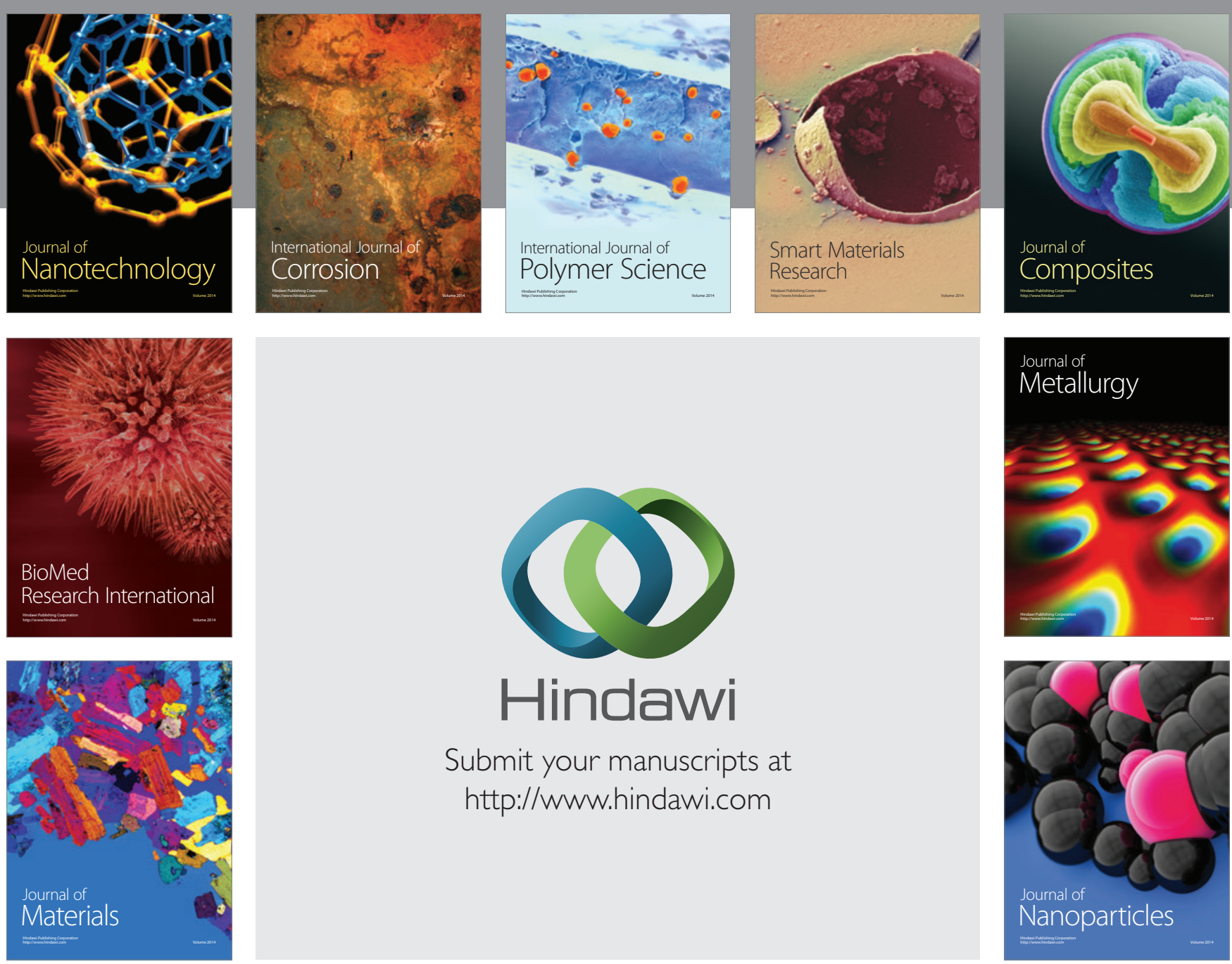

Submit your manuscripts at http://www.hindawi.com
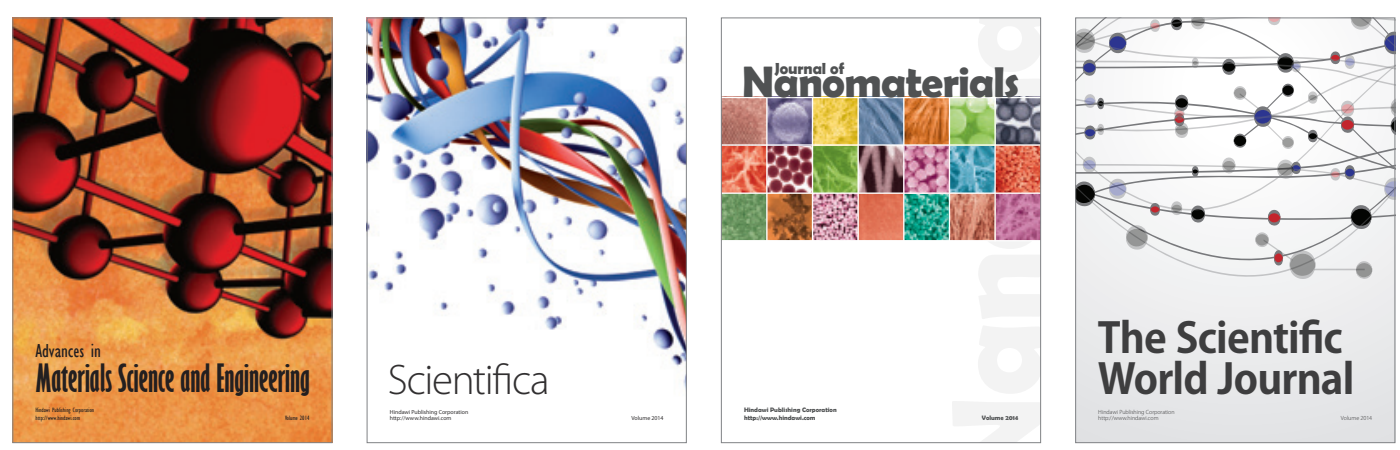

\section{The Scientific World Journal}
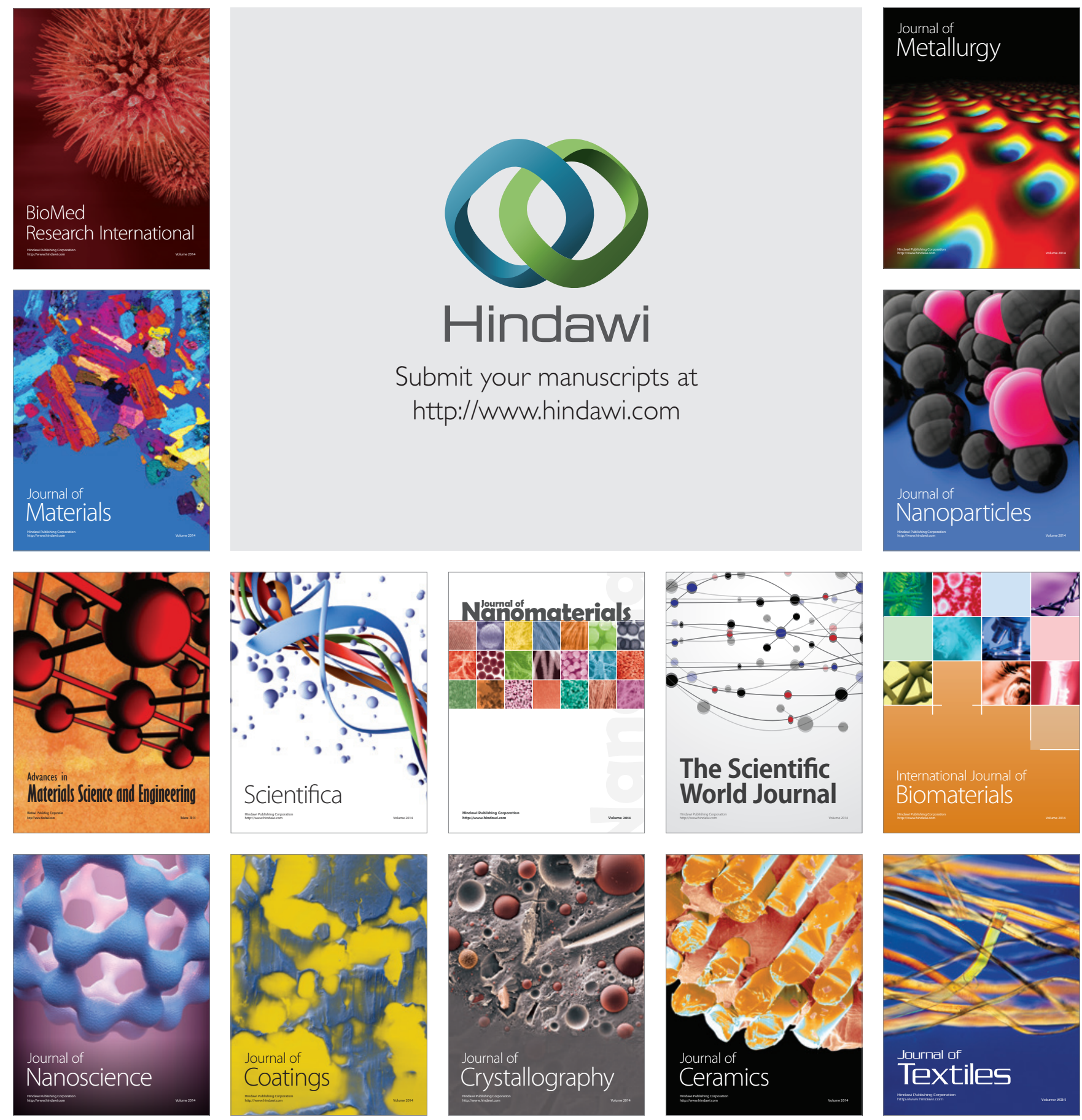